\title{
RINGS WITH ASSOCIATORS IN THE LEFT AND MIDDLE NUCLEUS
}

\author{
CHEN-TE YEN
}

\begin{abstract}
A.bstract. Let $R$ be a nonassociative ring, $N, M$ and $L$ the left, middle and right nucleus respectively. It is shown that if $R$ a semiprime ring satisfying $(R, R, R) \subset N \cap M$ (resp. $(R, R, R) \subset M \cap L)$, then $L \subset M \subset N$ (resp. $N \subset M \subset L)$ ); moreover, $R$ is associative if $((R, R, M),(R, R, R))=0$ (resp. $((M, R, R),(R, R, R))=0)$ or $(M, R) \subset$ $M$; and the Abelian group $(R,+)$ has no elements of order 2. We also prove that if $R$ is a simple ring satisfying char $R \neq 2$, and $(R, R, R) \subset$ $N \cap M$ or $(R, R, R) \subset M \cap L$ then $R$ is associative.
\end{abstract}

\section{Introduction}

Let $R$ be a nonassociative ring. We adopt the usual notation for associators and commutators: $(x, y, z)=(x y) z-x(y z),(x, y)=x y-y x$. We shall denote the left nucleus, middle nucleus, right nucleus and nucleus by $N, M, L$ and $G$ respectively. Thus $N, M, L$ and $G$ consists of all elements $n$ such that $(n, R, R)=0$, $(R, n, R)=0,(R, R, n)=0$ and $(n, R, R)=(R, n, R)=(R, R, n)=0$ respectively. A ring $R$ is called prime if the product of any two nonzero ideals is nonzero. $R$ is called serniprime if the only ideal of $R$ which squares to zero is the zero ideal. Kleinfeld [2] weakened Thedy's hypotheses [3] to obtain the following result: If $R$ is a prime ring which satisfies $(x, R, x) \subset N$ and $(R, R) \subset N$, then

Received November 1, 1991; revised May 9, 1992.

1980 Mathematics Subject Classification (1985 Revision).

Primary 17A.30.

Key words and phrases: Nonassociative ring, semiprime ring, prime ring, simple ring. 
$R$ is either associative or commutative. In the notes [4,5,6,7], we weaken Kleinfeld's hypotheses to obtain the same result. In [1], Kleinfeld proved that if $R$ is a semiprime ring satisfying $(R, R, R) \subset G$ and the Abelian group $(R,+)$ has no elements of order 2, then $R$ is associative. In [8], using Kleinfeld's result we improve his result as follows: If $R$ is a semiprime ring satisfying $(R, R, R) \subset N \cap L$, and the Abelian group $(R,+)$ has no elements of order 2 , then $R$ is associative. In [7], using his result we prove that if $R$ is a semiprime ring which satisfies $(R, R, R) \subset N$ and the Abelian group $(R,+)$ has no elements of order 2 and $(N, R) \subset N$ then $R$ is associative. In this note, using his result we show the following: If $R$ is a semiprime ring satisfying $(R, R, R) \subset N \cap M$ (resp. $(R, R, R) \subset M \cap L)$, then $L \subset M \subset N$ (resp. $N \subset M \subset L)$; moreover, $R$ is associative if $((R, R, M),(R, R, R))=0$ (resp. $((M, R, R),(R, R, R))=0)$ or $(M, R) \subset M$; and the Abelian group $(R,+)$ has no elements of order 2. We also prove that if $R$ is a simple ring satisfying $(R, R, R) \subset N \cap M$ or $(R, R, R) \subset M \cap L$; and char $R \neq 2$ then $R$ is associative.

\section{Results}

Let $R$ be a nonassociative ring. In every ring one may verify the identity

$$
(w x, y, z)-(w, x y, z)+(w, x, y z)=w(x, y, z)+(w, x, y) z
$$

Suppose that $n \in N$. Then with $w=n$ in (1) we obtain

$$
(n x, y, z)=n(x, y, z) \quad \text { for all } n \text { in } N .
$$

Assume that $m \in L$. Then with $z=m$ in (1) we get

$$
(w, x, y m)=(w, x, y) m \text { for all } m \text { in } L .
$$

As consequences of (1), (2) and (3), we have that $N, M$ and $L$ are associative subrings of $R$.

We assume that $R$ satisfies

$$
(R, R, R) \subset N \cap M
$$


Using (1) and (*) we have

$$
w(x, y, z)+(w, x, y) z \in N \cap M .
$$

Then with $y \in M$ in (4), we get

$$
(R, R, M) R \subset N \cap M .
$$

Combining (5) with (*), and using (2) we obtain

$$
(R, R, M)(R, R, R)=0 .
$$

And. with $x \in(R, R, M)$ in (1), and applying $(*)$ and (5) we get

$$
R(R, R, M) \subset N
$$

Thus with $z \in M$ in (4), and using (7) we obtain $(R, R, R) M \subset N$. Applying this, $(*)$ and (2) we have

$$
(R, R, R)(M, R, R)=0
$$

Then with $z \in L$ in (4), we get

$$
(R, R, R) L \subset N \cap M
$$

Definition 1. Let I be the associator ideal of $R$. I consists of the smallest ideal which contains all associators.

Note that I may be characterized as all finite sums of associators and right (or left) multiples of associators, as a consequence of (1). Hence we have

$$
I=(R, R, R)+(R, R, R) R=(R, R, R)+R(R, R, R)
$$

Then with $x \in(R, R, R)$ and $y \in L$ in (1), and using (*) and (9) we obtain $(R(R, R, R), L, R)=0$. By $(*)$ and $(10)$, this implies $(I, L, R)=0$. Thus it follows from this, $(*),(2)$ and $(10)$ that

$$
(R, R, R)(R, L, R)=0 .
$$


Definition 2. Let $B=\{b \in I:(R, R, R) b=0\}$.

Lemma 1. If $R$ is a ring satisfying $(R, R, R) \subset N \cap M$, then $B$ is an ideal of $R$ and $I \cdot B=0$.

Proof. Assume that $b \in B, x, y, z, w \in R$. Then using $(*)$, we have $(x, y, z)(b w)=((x, y, z) b) w=0$. Thus $b R \subset B$. On the other hand, applying $(*)$ twice and $(1)$ we obtain $(w, x, y)(z b)=((w, x, y) z) b=-(w(x, y, z)) b=$ $-w((x, y, z) b)=0$. Hence $R b \subset B$. At this point we have verified that $B$ is an ideal of $R$. Obviously, we get $(R, R, R) B=0$ and $((R, R, R) R) B=0$. Thus by (10) we obtain $I \cdot B=0$, as desired.

Lemma 2. If $R$ is a ring satisfying $(R, R, R) \subset N \cap M$ and $(R, R, R) N \subset$ $M$, then $(R, R, R)(R, N, R)=0$.

Proof. Assume that $(R, R, R) N \subset M$. Then with $x \in(R, R, R)$ and $y \in N$ in (1), and using (*) we obtain $(R(R, R, R), N, R)=0$. Applying this, $(*)$ and (10), we have $(I, N, R)=0$. Thus this implies $(R, R, R)(R, N, R)=0$, as desired.

Then with $w \in N$ in (4), we have

$$
N(R, R, R) \subset N \cap M
$$

Also with $x \in N$ in (4), we get $(R, N, R) R \subset N \cap M$. Using this, (*) and (2), we obtain

$$
(R, N, R)(R, R, R)=0
$$

Then with $x \in N$ and $y \in(R, R, R)$ in (1), and applying (*) and (12), we have

$$
(R, N,(R, R, R) R)=(R, N,(R, R, R)) R .
$$

Theorem 1. If $R$ is a semiprime ring satisfying $(R, R, R) \subset N \cap M$, then $G=L \subset M \subset N$; moreover, $R$ is associative if $((R, R, M),(R, R, R))=0$ or $(M, R) \subset M$; and the Abelian group $(R,+)$ has no elements of order 2. 
Proof. Using Lemma 1, we have $B^{2}=0$. Thus by semiprimeness of $R$, we obtain $B=0$. Applying (8) and (11), we get $(M, R, R) \subset B$ and $(R, L, R) \subset B$. Hence we have $(M, R, R)=(R, L, R)=0$, and so $G=L \subset M \subset N$.

Assume that $((R, R, M),(R, R, R))=0$. Then using this and (6) we obtain $(R, R, R)(R, R, M)=0$. Thus we get $(R, R, M) \subset B$ and so $(R, R, M)=0$. Hence $M=L=G$. Thus $(R, R, R) \subset G$. By Kleinfeld's result $[1], R$ is associative.

Assume that $M$ is a Lie ideal of $R$. Combining this with (12) yields $(R, R, R) N \subset M$. Applying Lemma 2, we have $(R, N, R) \subset B$ and so $(R, N, R)=$ 0 . Thus $N=M$. Hence $(R, R, R) \subset N$ and $(N, R) \subset N$. By Theorem 1 of [7], $R$ is associative.

Definition 3. Let $A=\{a \in I: a(R, R, R)=0\}$.

Recall that a ring $R$ is called simple if $R$ is the only nonzero ideal of $R$. Thus, a simple ring is prime if $R^{2} \neq 0$, and a prime ring is semiprime.

Theorem 2. If $R$ is a simple ring satisfying $(R, R, R) \subset N \cap M$, and char $R \neq 2$, then $R$ is associative.

Proof. By simplicity of $R$, we have $R^{2}=0$ or $R^{2}=R$; and $I=0$ or $I=R$. If $R^{2}=0$ or $I=0$, then $R$ is associative. Assume that $R^{2}=R$ and $I=R$.

Thus by (10) and (*), we have $R=R^{2}=((R, R, R)+(R, R, R) R) R=$ $(R, R, R) R+(R, R, R)\left(R^{2}\right)=(R, R, R) R$. Hence using this, (14) and (13), we obtain $(R, N, R)=(R, N,(R, R, R) R)=(R, N,(R, R, R)) R=$ $(R, N,(R, R, R))((R, R, R) R)=((R, N,(R, R, R))(R, R, R)) R=0$. Thus we have $N \subset M$. Combining this with Theorem 1 yields $N=M$. Then with $y \in N$ in (4), we obtain $(R, R, N) R \subset N$. Combining this with (*) yields $(R, R, N)(R, R, R)=0$. Hence $(R, R, N) \subset A$. Assume that $a \in A$ and $x \in R$. Then $a(R, R, R)=0$, and using $(*)$ we get $a R=a((R, R, R) R)=$ $(a(R, R, R)) R=0$. Thus $A=\{a \in R: a R=0\}$. Obviously, $A \subset N$. Because of $N=M$, we obtain $(a x) R=0$ and $(x a) R=x(a R)=0$. Hence $A$ is an ideal of $R$. By simplicity of $R$ and $R^{2}=R$, we have $A=0$. Thus, $(R, R, N) \subset A$ implies 
$(R, R, N)=0$. Hence $N=L=G$ by Theorem 1. Thus $(R, R, R) \subset G$. By Kleinfeld's result [1], $R$ is associative. This contradicts to $I=R$. Hence $I=0$. Therefore $R$ is associative.

By symmetry, we can prove the following:

Lemma 3. If $R$ is a ring satisfying $(R, R, R) \subset M \cap L$, then $A$ is an ideal of $R$ and $A \cdot I=0$.

Theorem 3. If $R$ is a semiprime ring satisfying $(R, R, R) \subset M \cap L$, then $G=N \subset M \subset L$; moreover, $R$ is associative if $((M, R, R),(R, R, R))=0$ or $(M, R) \subset M$; and the Abelian group $(R,+)$ has no elements of order 2 .

Theorem 4. If $R$ is a simple ring satisfying $(R, R, R) \subset M \cap L$, and char $R \neq 2$, then $R$ is associative.

Using the result of [4], we can improve the result of [5] as follows:

Theorem 5[5]. If $R$ is a prime ring with $N \neq 0$ satisfying $(x, y, z)+$ $(z, y, x) \in N$ and $(N+N R, R) \subset N$, then $R$ is either associative or commutative.

Added in proof. In [7], we have proved that if $R$ is a prime ring which satisfies $(x, y, z)+(z, y, x) \in N,(N, R) \subset N$ and $((R, R), R, R) \subset N$ then either $(N, R)=0$ or char $R=2$ or $R$ is associative. By adding the hypothesis " $(R, R), R, R) \subset N$ " in the Main Theorem of [5], we obtain Thedy's and Kleinfeld's results for the semiprime ring case. This result is in [7]: If $R$ is a semiprime ring which satisfies $(x, y, z)+(z, y, x) \in N,(N, R) \subset N,(N R, R) \subset N$ and $((R, R), R, R) \subset N$, then $R$ is a subdirect sum of a semiprime associative ring and a semiprime commutative ring.

\section{References}

[1] E. Kleinfeld, "A class of rings which are very nearly associative", Amer. Math. Monthly, 93 (1986), 720-722.

[2] E. Kleinfeld, "Rings with $(x, y, x)$ and commutators in the left nucleus", Comm. in Algebra, 16 (1988), 2023-2029.

[3] A. Thedy, "On rings with commutators in the nuclei", Math. Z., 119 (1971), 213-218. 
[4] C. T. Yen, "Rings with $(x, y, z)+(z, y, x)$ and $\left(N+R^{2}, R\right)$ in the left nucleus", unpublished manuscript.

[5] C. T. Yen, "Rings with $(x, R, x)$ and $(N+N R, R)$ in the left nucleus", Tamkang J. Math., 23 (1992), 247-251.

[6] C. T. Yen, "Rings with $(x, y, z)+(z, y, x)$ and $(N, R)$ in the left nucleus", submitted.

[7] C. T. Yen, "Rings with $(x, y, z)+(z, y, x),(N, R)$ and $((R, R), R, R)$ in the left nucleus", submitted.

[8] C. T. Yen, "Rings with associators in the left and right nucleus", submitted.

Department of Mathematics, Chung Yuan Christian University, Chung Li, Taiwan, 320, Republic of China. 